\title{
Numerical Convergence of the Random Vortex Method for Complex Flows
}

\author{
I. Mortazavi, P. Micheau \\ Laboratoire de mécanique de Lille \\ URA CNRS 1441 \\ Bd. Paul Langevin \\ 59650 Villeneuve d'Ascq, France
}

\author{
A. Giovannini \\ Laboratoire de modélisation en \\ mécanique des fluides de Toulouse \\ 118 route de Narbonne \\ 33602 Toulouse, France
}

\begin{abstract}
Vortex methods rely principally on a discretization of the continuous two-dimensional time dependent vorticity field into a large number of vortex "blobs", whose position and strength determine the underlying velocity field. In this paper, the convergence of the random vortex method (RVM) for a complex flow is studied in function of three discretization parameters. Two of these parameters are related to the spatial discretization of the vorticity, i.e. $\Gamma$ (sheet or blob strength) and $h$ (sheet length or core radius of a blob) and the third one to the discretization of time, i.e. $\Delta t$. Two principal events are observed. First, the computation works but the convergence is not attained. Second, the computation fails. The first behaviour is attributed to a lack of accuracy while the second is attributed to a lack of numerical stability. Once the stability conditions are satisfied, decreasing the value of the parameters always leads to convergence.
\end{abstract}

\section{Introduction}

Two-dimensional vortex methods rely principally on a discretization of the continuous two-dimensional time-dependent vorticity field into a large number of vortex "blobs" [5], whose position and strength determine the underlying velocity field [26]. These methods have gained considerable popularity in recent years and have been used in a variety of settings (see [6, 11, 17, 22, 27]). Some previous applications have focussed on predicting gross qualitative structures of turbulent flow $[3,10,13]$ and on their quantitative comparison with experiments [9, 21]. Currently, we have excellent theories for inviscid flows and a good understanding of the viscous flow provided there are no boundaries $[14,19]$. Benfatto and Pulvirenti [2] have presented interesting theoretical studies to provide the convergence of the random vortex method (RVM) with the creation of vorticity on solid boundaries. Furthermore, Puckett [24] has studied extensively the vortex sheet method and the consistency of the random walk method for the shear layer. On the other hand, numerical accuracy of the RVM has been provided by Sethian and Ghoniem [26] for complex flows with moderate and high Reynolds numbers.

In this paper, we present a detailed study of the numerical convergence of the random vortex method applied to a two-dimensional, viscous and incompressible flow. The goal is to analyze the effect of the choice of time step, vortex strength and core size on the computed solution. The test model is that of an internal jet seperated from an external flow by two bluff-body flat plates (Fig. 1). The present work complements the paper of Sethian and Ghoniem [26]. Sethian and Ghoniem had based their validation study principally on the physical comparison between numerical and 
experimental results for laminar and turbulent flows. In this work our aim is primarily to find a precision and stability zone, observing and analyzing the convergence of numerical results in function of $\Gamma, \Delta t$ and $h$; furthermore, this paper contains some combined parametric studies relating the influence of discretization parameters on each other.

\section{Numerical scheme}

The random vortex method is a numerical technique to solve the incompressible, 2D and unsteady Navier-Stokes equations converted to a rotational non-primitive formulation. Equations to be solved are: $\Delta \psi=-\omega$ and the vorticity transport equation (VTE): $\partial \omega / \partial t+\mathbf{u} \cdot \nabla \omega=(1 / R e) \Delta \omega$, associated with their boundary conditions. On the other hand, to discretize the vorticity, the computational domain is divided into two regions: the "interior" region and the numerical boundary region. In the interior region the vorticity is discretized using vortex "blobs" [5], while in the boundary domain vortex "sheets" are used [6]. The resolution technique of $\Delta \psi=-\omega$ is different for the two domains of discretization. Elsewhere, the VTE is solved by a two fractional step method: the first step corresponding to the convection of vorticity and the second one to its diffusion.

\subsection{The rotational non-primitive formulation}

\subsubsection{Velocity versus vorticity}

The continuity equation is satisfied using a streamfunction

$$
u=\frac{\partial \psi}{\partial y}, \quad v=-\frac{\partial \psi}{\partial x}
$$

where $\mathbf{u}=(u, v)$. The non-zero vorticity component normal to the flow plane is related to the velocity by

$$
\omega=\frac{\partial v}{\partial x}-\frac{\partial u}{\partial y}
$$

So the streamfunction is the solution of

$$
\Delta \psi=-\omega
$$

The solution of this equation in a free boundary domain is given by the Green function of the Poisson equation:

$$
\psi(\mathbf{x}, t)=\int_{D} G\left(\mathbf{x}-\mathbf{x}^{\prime}\right) \omega\left(\mathbf{x}^{\prime}\right) d \mathbf{x}^{\prime}
$$

where

$$
G(\mathbf{x})=-\frac{1}{2 \pi} \ln (r)
$$

and $r^{2}=x^{2}+y^{2}, d \mathbf{x}=d x \cdot d y ;$ then

$$
\mathbf{u}_{\omega}(\mathbf{x}, t)=\int_{D} \mathbf{K}\left(\mathbf{x}-\mathbf{x}^{\prime}\right) \omega\left(\mathbf{x}^{\prime}\right) d \mathbf{x}^{\prime},
$$

where $\mathbf{K}(\mathbf{x})=\frac{1}{2 \pi} \cdot \frac{(y,-x)}{r^{2}}$ is the integral kernel of the Poisson equation. 
In a bounded domain and for an inviscid flow, the solution is searched as: $\mathbf{u}=\mathbf{u}_{p}+\mathbf{u}_{\omega}, \mathbf{u}_{p}$ being the irrotational component of the velocity

$$
\mathbf{u}_{p}=\operatorname{grad}(\phi) \quad \text { and } \quad \Delta \phi=0,
$$

where $\phi$ is the potential function. The boundary conditions associated to (6) are such that $\mathbf{u}$ satisfies globally the boundary condition $\mathbf{u} \cdot \mathbf{n}=0$ on the wall, $\mathbf{u}$ given at the entrance of the domain and $\partial u / \partial x=0$ at the exit ( $\mathbf{n}$ is the normal unit vector to the wall).

For a viscous flow the wall condition is $\mathbf{u}=0$. Near the wall, in the boundary layer, since $\frac{\partial v}{\partial x} \ll \frac{\partial u}{\partial y}$, Eq. 2 is reduced to

$$
\omega=-\frac{\partial u}{\partial y}
$$

where $x$ and $y$ correspond respectively to parallel and normal directions at solid boundaries. Then we obtain

$$
u(x, y, t)=u_{\infty}(x, t)+\int_{y}^{\infty} \omega\left(x, y^{\prime}, t\right) d y^{\prime}
$$

and, using the continuity equation:

$$
v(x, y, t)=-\int_{0}^{y} \frac{\partial u}{\partial x}\left(x, y^{\prime}, t\right) d y^{\prime}
$$

while $u_{\infty}(x, t)$ is the $x$-component of $\mathbf{u}$ on the edge of the boundary layer.

\subsubsection{The vorticity transport equation}

The momentum equations in vortical and $2 \mathrm{D}$ formulation are expressed by the VTE, i.e.

$$
\frac{\partial \omega}{\partial t}+\mathbf{u} \cdot \nabla \omega=\frac{1}{R e} \Delta \omega
$$

Furthermore, the VTE in Lagrangian form can be written as

$$
\frac{D \omega}{D t}(\mathbf{x}, t)=\frac{1}{R e} \Delta \omega .
$$

This equation is directly associated to

$$
\frac{d \mathbf{x}}{d t}=\mathbf{u}
$$

This last equation yields:

$$
\mathbf{x}(\boldsymbol{\alpha}, t)=\boldsymbol{\alpha}+\int_{0}^{t} \mathbf{u} \cdot d t
$$

with $\mathbf{x}(\boldsymbol{\alpha}, 0)=\boldsymbol{\alpha}$.

Equation 11 is solved by a two fractional steps method. First, we solve the convection part of this equation using (12) and $D \omega(\mathbf{x}, t) / D t=0$ :

$$
\frac{\partial \omega}{\partial t}+\mathbf{u} \cdot \nabla \omega=0
$$


where $\omega$ is constant along the trajectory of a particle.

The second step is devoted to diffusion:

$$
\frac{\partial \omega}{\partial t}=\frac{1}{R e} \Delta \omega,
$$

that is solved using the random walk method [12]. These two solutions are combined to obtain the total transport of vorticity due to convection and diffusion. Beale and Majda [1] have proven that viscous splitting algorithms converge to the solution of Navier-Stokes equations in all of space, at a rate which increases as the viscosity decreases.

\subsection{Discretization procedure}

\subsubsection{Velocity versus vorticity}

In the interior domain, we discretize $\omega(\mathbf{x})$ by small area elements called vortex blobs. Each element carries a finite and invariant circulation $\Gamma$. The vorticity of a blob is distributed according to a core function $f_{\delta}(\mathbf{r})$ with a finite core radius $\delta$. The vorticity within the core is smooth and the velocity in the center of elements is finite. Then, the vorticity can be approximated by

$$
\omega(\mathbf{x})=\sum_{i=1}^{N V} \Gamma_{i} f_{\delta}\left(\mathbf{x}-\mathbf{x}_{i}\right),
$$

where $N V$ is the number of vortex blobs; $f_{\delta}$ is a rapidly decreasing, radially symmetric core smoothing function such that

$$
\int_{R^{2}} f(\mathbf{r}) d \mathbf{r}=1
$$

Furthermore

$$
f_{\delta}(\mathbf{r})=\frac{1}{\delta^{2}} f_{\delta}\left(\frac{\mathbf{r}}{\delta}\right),
$$

where $\delta$ is the core radius. As $\delta \rightarrow 0, f_{\delta}$ approaches the Dirac delta function. So, using Eq. 5, the rotational velocity is approximated by

$$
\mathbf{u}_{\omega}\left(x_{j}, y_{j}\right)=\sum_{i=1}^{N V} \Gamma_{i} \mathbf{K}_{\delta}\left(\mathbf{x}_{j}-\mathbf{x}_{i}\right)
$$

where

$$
\begin{gathered}
\mathbf{K}_{\delta}\left(\mathbf{x}, \mathbf{x}_{i}\right)=\int \mathbf{K}\left(\mathbf{x}-\mathbf{x}^{\prime}\right) \cdot f_{\delta}\left(\mathbf{x}^{\prime}-\mathbf{x}_{i}\right) d \mathbf{x}^{\prime} \\
\mathbf{K}_{\delta}(\mathbf{x}, 0)=-\frac{1}{2 \pi} \cdot \frac{(y,-x)}{r^{2}} k_{\delta}\left(\frac{r}{\delta}\right) \\
k_{\delta}\left(\frac{r}{\delta}\right)=\frac{2 \pi}{\delta^{2}} \int_{0}^{r} r f_{\delta}\left(\frac{r}{\delta}\right) d r .
\end{gathered}
$$

In this work a Rankine core function is used to smooth the velocity [21], i.e.

$$
\begin{array}{clll}
f_{\delta}\left(\frac{r}{\delta}\right)=\frac{1}{\pi} & \text { and } & k_{\delta}\left(\frac{r}{\delta}\right)=\frac{r^{2}}{\delta^{2}}, & \text { if } \frac{r}{\delta} \leq 1 \\
f_{\delta}\left(\frac{r}{\delta}\right)=0 & \text { and } & k_{\delta}\left(\frac{r}{\delta}\right)=1, & \text { if } \frac{r}{\delta}>1 .
\end{array}
$$


Other choices for core functions have been suggested by Chorin [5], Beale and Majda [1] and Cottet [7]. The performance and efficiency of these cutoffs have been studied by Beale and Majda [1] and Perlman [23]. On the other hand, a new version of the well-known point vortex method is used in modern vortex methods. Goodman et al. [15] proved that this point vortex method converges for all times if the flow is smooth while Cottet [8] demonstrated their corresponding stability conditions. However, using core functions is more than a mathematical need [16]: it yields smaller errors for long time, higher order of convergence for smooth flows and allows pointwise evolution of velocity and vorticity.

In this work a finite element method is used to solve the equation

$$
\Delta \phi=0
$$

at each time step with boundary conditions: $\operatorname{grad} \phi \cdot \mathbf{n}=\mathbf{u}_{p} \cdot \mathbf{n}=-\mathbf{u}_{\omega} \cdot \mathbf{n}$. The solution is obtained, assuming linear basis functions in triangular elements for the approximation of $\phi$, and a Galerkin weighted residual minimization to approximate the differential equation by a set of linear algebric equations [21]. This method seems to be convenient and flexible to apply in complex flow geometries.

The potential velocity being defined by

$$
\mathbf{u}_{p}=\left\{-\frac{\partial \phi}{\partial x}, \frac{\partial \phi}{\partial y}\right\}
$$

and discrete potential velocity being constant for each element. To obtain $\mathbf{u}_{p}$ at the center of the vortex blob or the vortex sheet (defined below) it is necessary to "interpolate" the potential velocity value, if the vortex elements center is located on the boundaries or nodes of finite elements.

As shown in section (2.1.2), the velocity in the boundary layer is almost parallel to the wall and must be zero on the wall. With the vortex blob method, it is not possible to satisfy these conditions.

Therefore, the vorticity in the boundary layer is descretized by vortex sheets [6]. The vorticity at each time step is approximated by a sum of linear concentrations of vorticity on segments:

$$
\tilde{\omega}(x, y)=\sum_{j} \gamma_{j} b_{h}\left(x-x_{j}\right) \delta\left(y_{j}-y\right)
$$

Each term of this sum is refered as a vortex sheet. The $j$ th sheet's center and strength (or weight) are respectively represented by $\left(x_{j}, y_{j}\right)$ and $\gamma_{j}$. In Eq. $26, \delta$ is the Dirac delta function and $b_{h}=b(x / h)$ is what we refer to as the smoothing or cutoff function in analogy with the vortex method. A constant variable core function is used in this work:

$$
b_{h}(x)= \begin{cases}1 & \text { for }\left|\frac{2 x}{h}\right| \leq 1 \\ 0 & \text { otherwise }\end{cases}
$$

with $h$ as the sheet length and $\gamma_{i}=\Gamma_{i} / h$. Other cutoff functions are proposed in [24].

The vortex sheet is transformed to a vortex blob as it crosses the numerical boundary layer and the same procedure occurs inversely in the opposite way. To assume the continuity of $\omega$ through the boundary layer, the sheet length is related to the core radius by: $\delta=h / 2$. The "sheet" boundary layer thickness (which is not exactly the same as the physical boundary layer) is proportional to $\sqrt{\Delta t / R e}$, i.e. the temporal growth of the boundary layer during time.

The approximation for $u$ is determined using Eq. 8 and Eq. 9:

$$
\tilde{u}(x, y)=u_{\infty}(x)+\sum_{j=1}^{N V} \gamma_{j} b_{h}\left(x-x_{j}\right) H\left(y_{j}-y\right),
$$


where $H(y)$ is the Heaviside function:

$$
H(y)= \begin{cases}1 & \text { for } y \geq 0 \\ 0 & \text { otherwise }\end{cases}
$$

The velocity jump along the $j$ th sheet is therefore equivalent to $\gamma_{j} b_{h}\left(x-x_{j}\right)$. For the normal component of the velocity we can write

$$
\tilde{v}(x, y)=-\frac{\partial u_{\infty}(x) y}{\partial x}-\frac{1}{h} \sum_{j=1}^{N V} \gamma_{j}\left[b_{h}\left(x^{+}-x_{j}\right)-b_{h}\left(x^{-}-x_{j}\right)\right] \cdot \min \left(y, y_{j}\right),
$$

where $x^{+}=x+h / 2$ and $x^{-}=x-h / 2$ while

$$
u_{\infty}(\mathbf{x})=\mathbf{u}_{\omega}\left(x, \delta_{S}\right)+\mathbf{u}_{p}\left(x, \delta_{S}\right),
$$

where $\delta_{S}$ is the sheet layer thickness.

At each time $k \cdot \Delta t$, on each grid point of the wall the number of newly created sheets must be convenient to annihilate the slip velocity at this point.

\subsubsection{The vorticity transport equation (VTE)}

The mathematical scheme for the convection and diffusion calculations in the boundary and interior domains are the same (the viscous splitting method) and the princpal difference lies in the vorticity discretization procedure (blob or sheet) that depends on the cutoff choice. Vortex sheets separate initially from the wall only by normal random displacements and in the whole boundary domain random displacements remain normal to the wall. An important number of papers are devoted to describe this procedure, e.g. Ghoniem and Gagnon [11] and Puckett [24]. Finally, the time integration scheme in the interior domain is the Heun's second order scheme, but in the boundary zone it is a simple first order Euler's scheme.

Solving Eq. 14 in a domain $D$ with boundaries $\partial D$ according to Eqs. 12 and 13, is equivalent to the statement that vorticity is convected by its own total velocity field $\mathbf{u}=\mathbf{u}_{p}+\mathbf{u}_{\omega}$. Thus, the vortex elements are moved by

$$
\frac{d \mathbf{x}_{j}}{d t}=\mathbf{u}_{\omega, j}(t)+\mathbf{u}_{p, j}(t)
$$

For the time integration of particles trajectories, Eq. 13, we use the modified Euler (Heun) method [21]. The evolution of computations due to the convection during the time step $\Delta t$ using this method can be described by

$$
\mathbf{x}_{j, \text { conv }}^{*}=\mathbf{x}_{j}^{k}+\Delta t\left(\mathbf{u}_{\omega, j}^{k}+\mathbf{u}_{p, j}^{k}\right)
$$

which corresponds to the predicted value of the trajectory; the corrected value in this equation is represented by

$$
\mathbf{x}_{j, \mathrm{conv}}^{k+1}=\mathbf{x}_{j}^{k}+\left(\frac{\Delta t}{2}\right)\left[\left(\mathbf{u}_{\omega, j}^{k}+\mathbf{u}_{p, j}^{k}\right)+\left(\mathbf{u}_{\omega, j}^{*}+\mathbf{u}_{p, j}^{*}\right)\right]
$$

where $\mathbf{x}_{j}^{k}=\left[x_{j}(k \Delta t), y_{j}(k \Delta t)\right]$ and $\mathbf{u}_{\omega, j}^{*}=\mathbf{u}_{\omega}\left(\mathbf{x}_{j}^{*}\right)$. This technique is second-order accurate. Such accuracy is desirable because as vortex blobs come close together they spin around each other. The 
accuracy of Heun's method compared to other time integrating techniques, with their implementation to particle methods is studied by Lucquin-Desreux [20] and Chien Chen Chang [4].

On the other hand, in the boundary region, as the curvature of the trajectories is not important, an Euler's first order method is used for time integration. Each sheet is moved by

$$
\mathbf{x}_{j, \text { conv }}^{k+1}=\mathbf{x}_{j}^{k}+\Delta t \mathbf{u}_{j}^{k}
$$

where $\mathbf{u}_{j}^{k}$ is defined by Eqs. 28 and 30 .

The random walk algorithm [12] is used to approximate Eq. 16. This method is desirable because we want to provide a Lagrangian approach to the heat equation. This is based on the rule that an infinite collection of particles undergoing Brownian motion yields a solution of the heat equation [25]. The 1D Green function of the diffusion equation is

$$
G r(y, t)=\sqrt{\frac{1}{4 \pi \nu t}} \exp \left(-\frac{1}{4 \pi \nu t} y^{2}\right)
$$

Equation 32 is identical to the probability density function of a Gaussian random variable $\eta$ with a zero mean and a standard deviation $\sigma=\sqrt{2 \nu t}$ :

$$
P(\eta, t)=\sqrt{\frac{1}{2 \pi \sigma^{2}}} \exp \left(-\frac{1}{2 \pi \sigma^{2}} \eta^{2}\right) .
$$

This solution can easily be extended in two dimensions:

$$
G r^{\prime}(x, y, t)=G r(x, t) G r(y, t) .
$$

The corresponding two-dimensional probability density function is expressed by

$$
P^{\prime}\left(\eta_{x}, \eta_{y} ; t\right)=P_{1}\left(\eta_{x} ; t\right) P_{2}\left(\eta_{y} ; t\right),
$$

thus Eq. 15 is solved stochastically by a 2D displacement of vortex blobs in two perpendicular directions, using a set of independent Gaussian random numbers. For more information on the random walk method and its consistency and stability, the reader is refered to Ghoniem and Sherman [12], Long [19] and Puckett [24].

Finally, the total transport of vortex blobs is obtained adding the two fractional movements

$$
\mathbf{x}_{j}^{k+1}=\mathbf{x}_{j, \mathrm{conv}}^{k+1}+\boldsymbol{\eta}_{j} \quad \text { with } \quad \boldsymbol{\eta}=\left(\eta_{x}, \eta_{y}\right) .
$$

As mentioned above, in the boundary domain the numerical solution of the diffusion is approximated letting all sheets undergo a random walk only in the $y$ direction and reflecting those that go below the boundary. Therefore the new sheet position at time $(k+1) \Delta t$ is

$$
\left(x_{j}^{k+1}, y_{j}^{k+1}\right)=\left(x_{j, \mathrm{conv}}^{k+1},\left|y_{j, \mathrm{conv}}^{k+1}+\eta_{y j}\right|\right) .
$$

Furthermore, newly created vortex sheets diffuse perpendicular to the wall without any convective motion.

The post-processing procedure to obtain final numerical results, i.e. streamfunction and velocity from the primary calculated parameters, the number of blobs $N V$, their circulation and their position $\left(x_{i}, y_{i}\right)$, is done using the vortex-in-cell method [18]. 


\section{Convergence and stability}

Numerical parameters used in the discretization of the equations and studied here, are:

1. the circulation of a vortex element, sheet or blob, $\Gamma$;

2. the sheet length, $h$, or core radius, $\delta(\delta=h / 2)$;

3. the time step value, $\Delta t$.

The two first parameters are used for the discretization of $\omega$ and the last one is the time discretization parameter.

In this work we are interested in studying the influence of these discretization parameters on the approximation of the VTE and the satisfaction of boundary conditions on solid boundaries. We suppose that calculation errors related to the irrotational flow computation and $\mathbf{u}_{\omega}$ (when $\omega$ is defined) are not crucial. Furthermore, we assumed that the post-processing technique (vortexin-cell) does not generate significant extra errors. The study is based on the evolution of averaged results on time, such that $F(t)=\left[1 /\left(t_{2}-t_{1}\right)\right] \int_{t_{1}}^{t_{2}} f(t) d t$. The averaging procedure starts once the asymptotical state is reached with a quasi-constant number of vortex elements. The main results obtained are presented in the following.

Figures 2 and 3 show the evolution of averaged streamlines and averaged streamwise velocity profiles (for a section downstream of the bluff-body at a distance equal to one from the bluffbody) as a function of varying $\Gamma$ values for $\Delta t=0.05$ and $h=0.05$. The averaging is done for the established flow on 500 successive steps. As shown in Fig. 2, for $\Gamma=0.017,0.008$ and 0.006 , the center of the recirculation zone is not well defined and contours around the large eddy downstream of the bluff-body are not similar. However, decreasing the circulation value to 0.0042 and 0.0033 , the convergence of this recirculation zone towards identical large eddy formation is observed. Streamlines have similar alignement and contours are smoother around the eddy. Figure 3 confirms the convergence of numerical results, when we decrease the vortex strength parameter. Small $\Gamma$ values imply an increasing number of blobs, so not only a "refined" discretization of vorticity with a sensible increase of the accuracy but also a better approximation of diffusion by the random walk technique. Furthermore, decreasing $\Gamma$ allows to have a better satisfaction of the no-slip boundary conditions.

Nevertheless, for a smaller value of $\Gamma(\Gamma=0.0027)$, in the beginning of the establishment of the flow, the computational procedure failed. Just before that failure the number of created vortex elements was strongly oscillating. The global time integration scheme is of explicit type and this behaviour is apparently due to numerical instabilities related to the time discretization of boundary conditions at the wall: $u=0$. When decreasing the time step value to 0.025 , fluctuations diminnished and results were quite satisfactory (Fig. 3). Therefore, we can deduce that a concomitant choice of these discretization parameters is necessary to avoid the onset of numerical instabilities when decreasing their values.

Figures 4 and 5 are obtained for constant values of $\Gamma=0.00417$ and $h=0.05$, and variable values of $\Delta t$. We observe the optimum results (for both averaged streamlines and velocity profiles) for $\Delta t=0.05$ and 0.035 (which means the convergence is attained when decreasing the time step value). However, for $\Delta t=0.025$ the solution diverges again. Apparently, for this case, the error in the approximation of the diffusion part of the VTE overshadows the gain of accuracy for the convection part $[20,26]$. Nevertheless, decreasing $\Gamma$ to 0.0031 , we observe that, along with the natural increase in the number of elements, we obtain a satisfactory solution. Figure 5 confirms the above improvement. 
Figures 6 and 7 show the influence of the sheet length on the calculations, $\Gamma$ and $\Delta t$ being constant (0.0042 and 0.05, respectively). The principal feature is that for $h=0.025$, the computational procedure is stopped because blobs left the domain at the beginning of the calculations. This feature corresponds to a local stability condition of the type "Courant" that can be written as $\Delta t \cdot \Gamma / h \leq \beta h,(\Delta t \cdot \Gamma) \leq \beta h^{2}$, where $\beta$ is a constant. We have verified that decreasing the time step value, i.e. $\Gamma=0.0042, \Delta t=0.025$ and $h=0.025$, gives a more stable solution, but less correct than the results with greater values of $h$ (Fig. 7). This fact is, likely, due to the discretization of $\omega$ which is related to $\Gamma / h^{2}$. Diminishing $\Gamma(\Gamma=0.0027, \Delta t=0.025, h=0.025)$, we obtain a converged solution (Fig. 8).

\section{Conclusion}

The convergence of the RVM for a complex flow in function of three discretization parameters is studied. Two of these parameters are related to the spatial discretization of the vorticity ( $\Gamma$ and $h$ ) and the third one to the discretization of time. Two principal events are observed: first, the computation works but the convergence is not attained; second, the computation fails. The first behaviour is attributed to a lack of accuracy and the second to a lack of numerical stability.

Two kinds of stability conditions are supposed: one of the "Courant" type, $(\Delta t \cdot \Gamma) \leq \beta h^{2}$; the other one related to the satisfaction of the boundary condition, $u=0$. To our knowledge, for the moment, no complete theory has been developped to express this condition. Anyway, once the stability conditions are satisfied (which depends primarily on a convenient choice of discretization parameters compared to each other), decreasing the value of parameters always lead to convergence. Additionally, we have observed a divergence of the numerical solution for a very small choice of the time step. This error, which is due to the random walk method, is diminished by generating a higher number of elements in the computational domain.

Finally, for some computations, we have verified the convergence behaviour of instantaneous results under the criterium of monitoring convergence values.

\section{Acknowledgements}

The authors are thankful to the second reviewer for his very helpful comments. All calculations were performed on a Cray C98 at "IDRIS-CNRS" (Orsay, France).

\section{References}

[1] Beale, T. and Majda, A., "High Order Accurate Vortex Methods with Explicit Velocity Kernels," J. Comp. Phys., 58, pp. 188, 1985.

[2] Benfatto, G. and Pulvirenti, M., "Generation of Vorticity near the Boundary in Planar NavierStokes Flows," Comm. Math. Phys., 96, pp. 59, 1984.

[3] Blot, F., Giovannini, A., Hebrard, P. and Strzelecki, A., "Flow Analysis in a Vortex Flowmeter: An Experimental and Numerical Approach," Turbulent Shear Flow, Stanford, 1989.

[4] Chang, C., "Random Vortex Methods for the Navier-Stokes Equations," J. Comp. Phys., 76, pp. 281, 1988.

[5] Chorin, A.J., "Numerical Study of Slightly Viscous Flow," J. Fluid Mech., 57, pp. 785, 1973. 
[6] Chorin, A.J., "Vortex Sheet Approximation of Boundary Layers," J. Comp. Phys., 27, pp. 428, 1978.

[7] Cottet, G.H., "A New Approach to the Analysis of Vortex Methods in Two and Three Dimensions," Ann. Inst. H. Poincaré Anal. Non Lin., 5, pp. 277, 1988.

[8] Cottet, G.H., "A Remark on the Stability of the Point Vortex Method," University of California, Internal Report, Los Angeles, CA, 1989.

[9] Gagnon, Y., Giovannini, A. and Hebrard, P., "Numerical Simulation and Physical Analysis of High Reynolds Number Flows behind Sudden Expansions," Phys. Fluids, A (5), pp. 2377, 1993.

[10] Ghoniem, A.F., Chorin, A.J. and Oppenheim, A.K., "Numerical Modeling of Turbulent Flow in a Combustion Tunnel," Phil. Trans. R. Soc. London, A (304), pp. 303, 1982.

[11] Ghoniem, A.F. and Gagnon, Y., "Vortex Simulation of Laminar Recirculating Flow," J. Comp. Phys., 68, pp. 346, 1987.

[12] Ghoniem, A.F. and Sherman, F.S., "Grid Free Simulation of Diffusion using Random Walk Methods," J. Comp. Phys., 61, pp. 1, 1985.

[13] Giovannini, A., Mortazavi, I. and Tinel, Y., "Numerical Flow Visualization in High Reynolds Numbers using Vortex Method Computational Results," ASME FED, 218, pp. 37, 1995.

[14] Goodman, J., "Convergence of the Random Vortex Method," Comm. Pure Appl. Math., 60, pp. 189, 1987.

[15] Goodman, J., Hou, T.Y., and Lowengrub, J., "The Convergence of the Point Vortex Method for the 2D Euler Equations," Comm. Pure and Appl. Math., 43, pp. 415, 1990.

[16] Hald, O.H., "Convergence of Vortex Methods," SIAM: Vortex Methods and Vortex Motion, Edited by K.E. Gustafson and J.A. Sethian, 33, 1991.

[17] Knio, O. and Ghoniem, A.F., "Three Dimensional Vortex Simulation of Rollup and Entrainment in a Shear Layer," J. Comp. Phys., 97, pp. 273, 1991.

[18] Leonard, A., "Vortex Methods for Flow Simulation," J. Comp. Phys., 37, pp. 289 (1980).

[19] Long, D.G., "Convergence of the Random Vortex Method in Two Dimensions," J. Amer. Math. Soc., 1, pp. 779, 1988.

[20] Lucquin-Desreux, B., "Particle Approximation of the Two-Dimensional Navier-Stokes Equations," Rech. Aérosp., 4, pp. 1, 1987.

[21] Martins, L.F. and Ghoniem, A.F., "Simulation of the Nonreacting Flow in a Bluff-Body Burner: Effect of the Diameter Ratio," J. Fluids Eng., 115, pp. 474, 1993.

[22] McCracken, M.F. and Peskin, C.S., "A Vortex Method for Blood Flow through Heart Valves," J. Comp. Phys., 35, pp. 183, 1980.

[23] Perlman, M., "On the Accuracy of Vortex Methods," J. Comp. Phys., 59, pp. 200, 1985.

[24] Puckett, E.G., "A Study of the Vortex Sheet Method and its Rate of Convergence," SIAM: J. Sci. Stat. Comp., 10, pp. 298, 1989. 
[25] Sethian, J., "A Brief Overview of Vortex Methods," SIAM: Vortex Methods and Vortex Motion, Edited by K.E. Gustafson and J.A. Sethian, 1, 1991.

[26] Sethian, J.A. and Ghoniem, A.F., "Validation Study of Vortex Methods," J. Comp. Phys., 74, pp. 283, 1988.

[27] Summers, D.M., Hanson, T. and Wilson, C.B., "A Random Vortex Simulation of Wind Flow over a Building," J. Num. Methods Fluid Mech., 5, pp. 849, 1985.

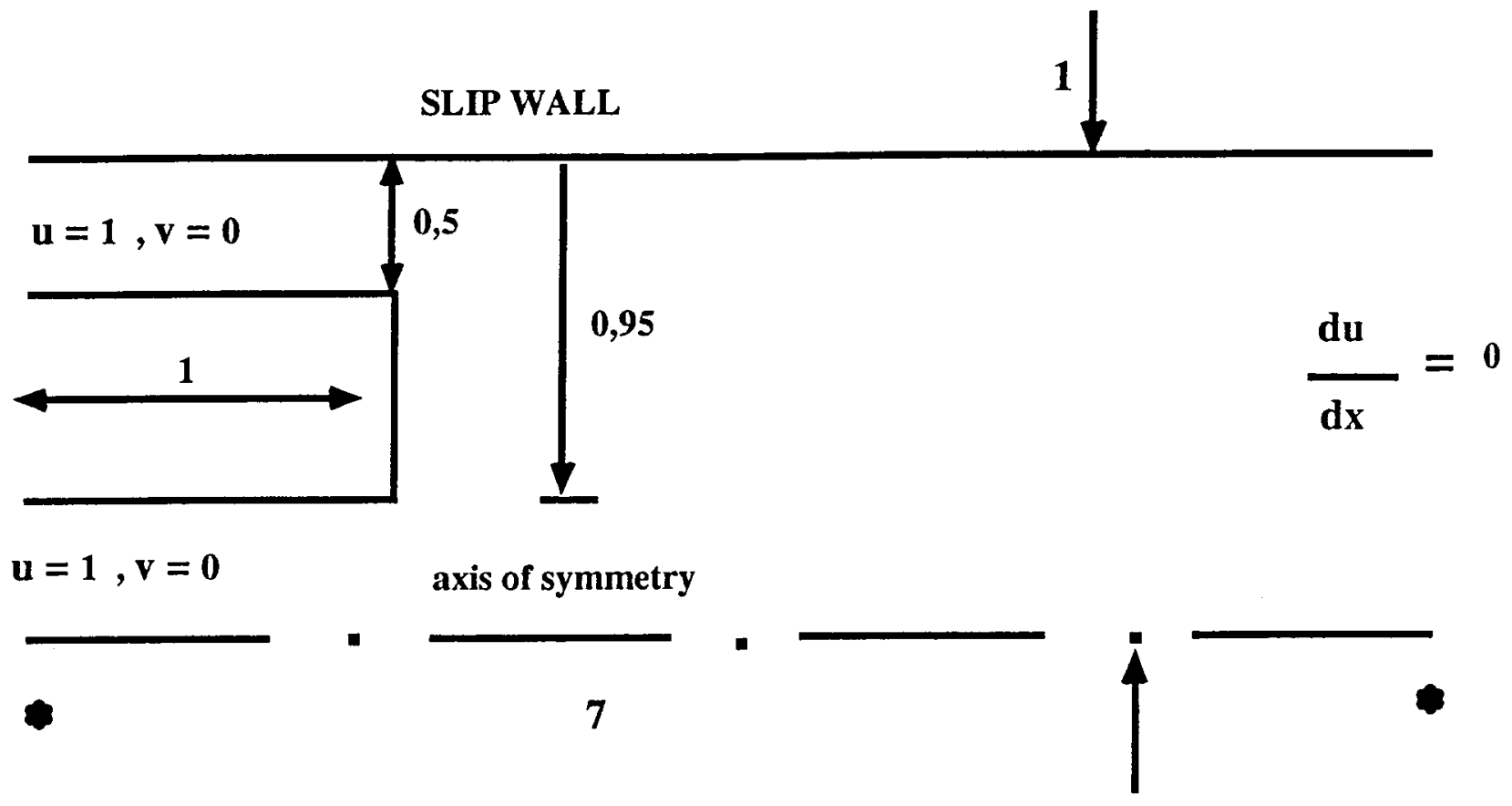

Figure 1: The geometry of the computational domain with inflow and outflow boundary conditions. 

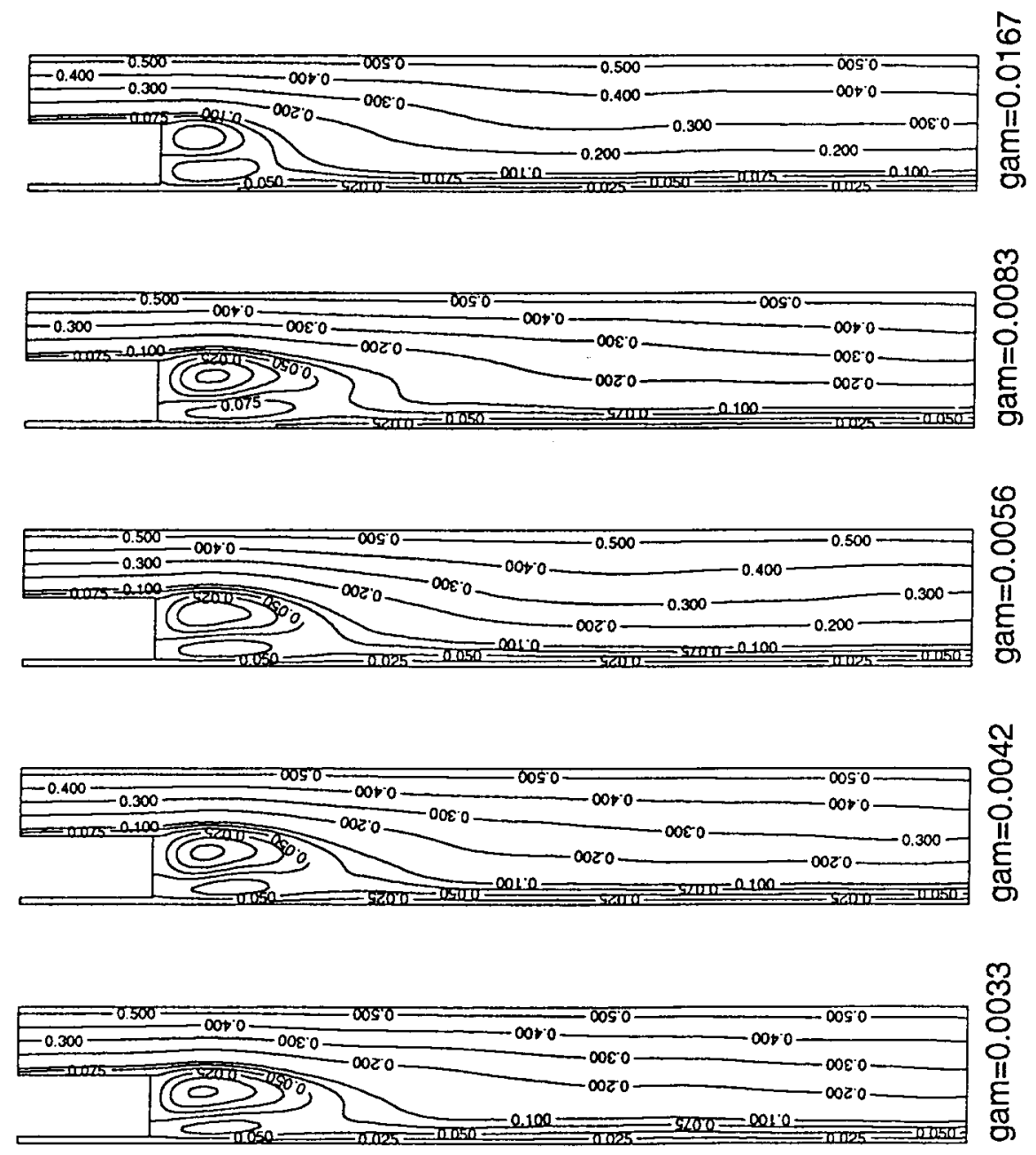

Figure 2: Average streamlines $(R e=7000, \Delta t=0.05, h=0.05$ for different values of $\Gamma)$. 


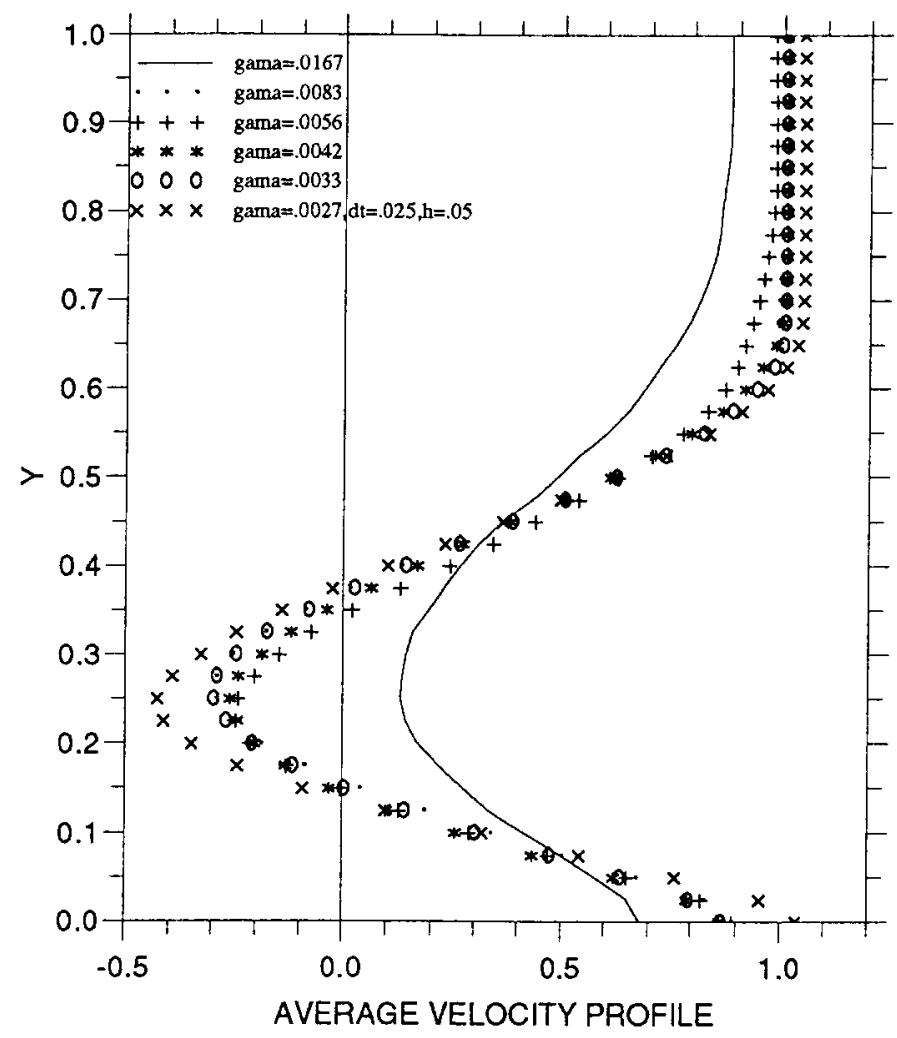

Figure 3: Average velocity profiles for $x=2$. $(R e=7000, \Delta t=0.05, h=0.05$ for different values of $\Gamma)$. 

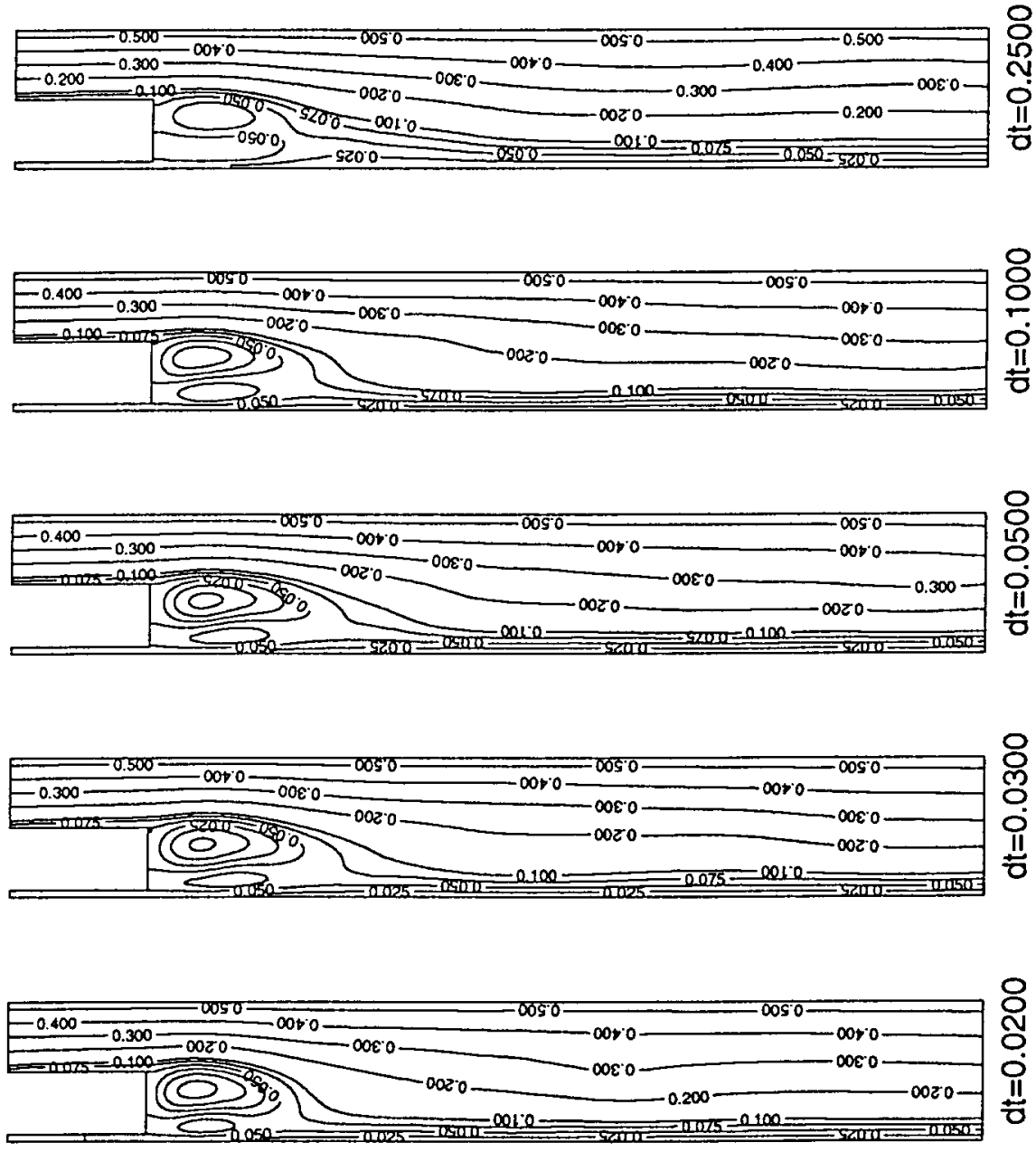

Figure 4: Average streamlines $(R e=7000, \Gamma=0.00417, h=0.05$ for different values of $\Delta t)$. 


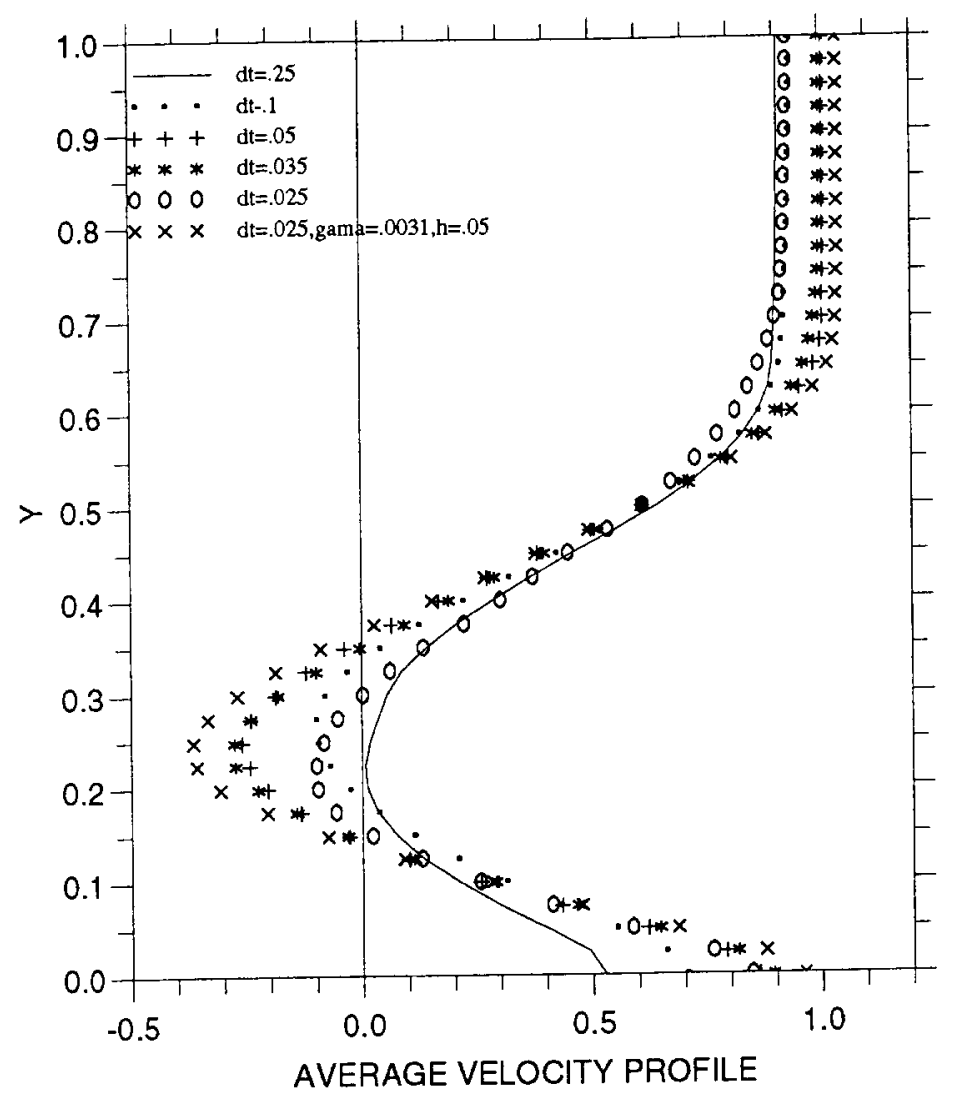

Figure 5: Average velocity profiles for $x=2 . \quad(R e=7000, \Gamma=0.00417, h=0.05$ for different values of $\Delta t$ ). 

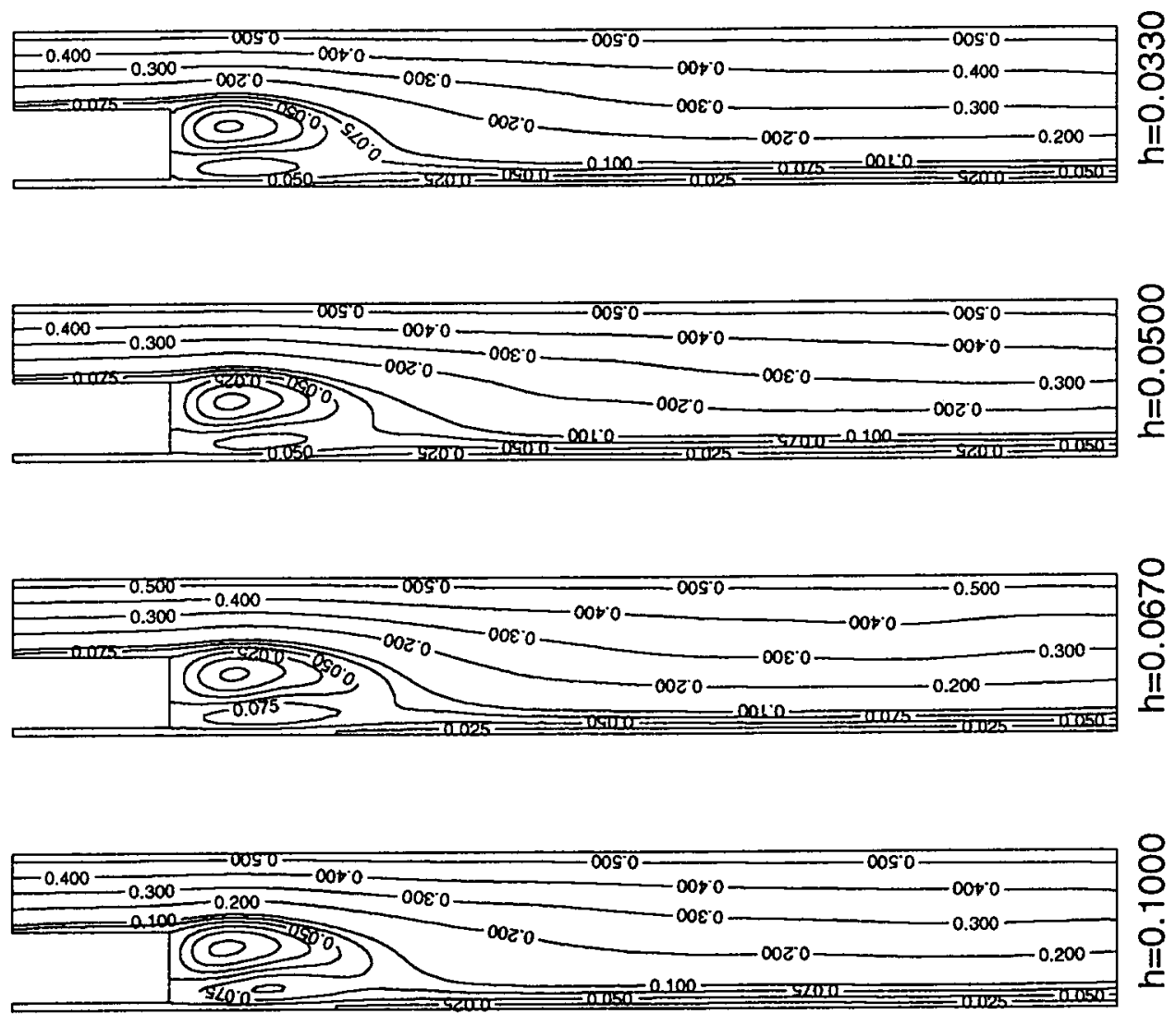

Figure 6: Average streamlines $(R e=7000, \Gamma=0.00417, \Delta t=0.05$, for different values of $h$ ). 


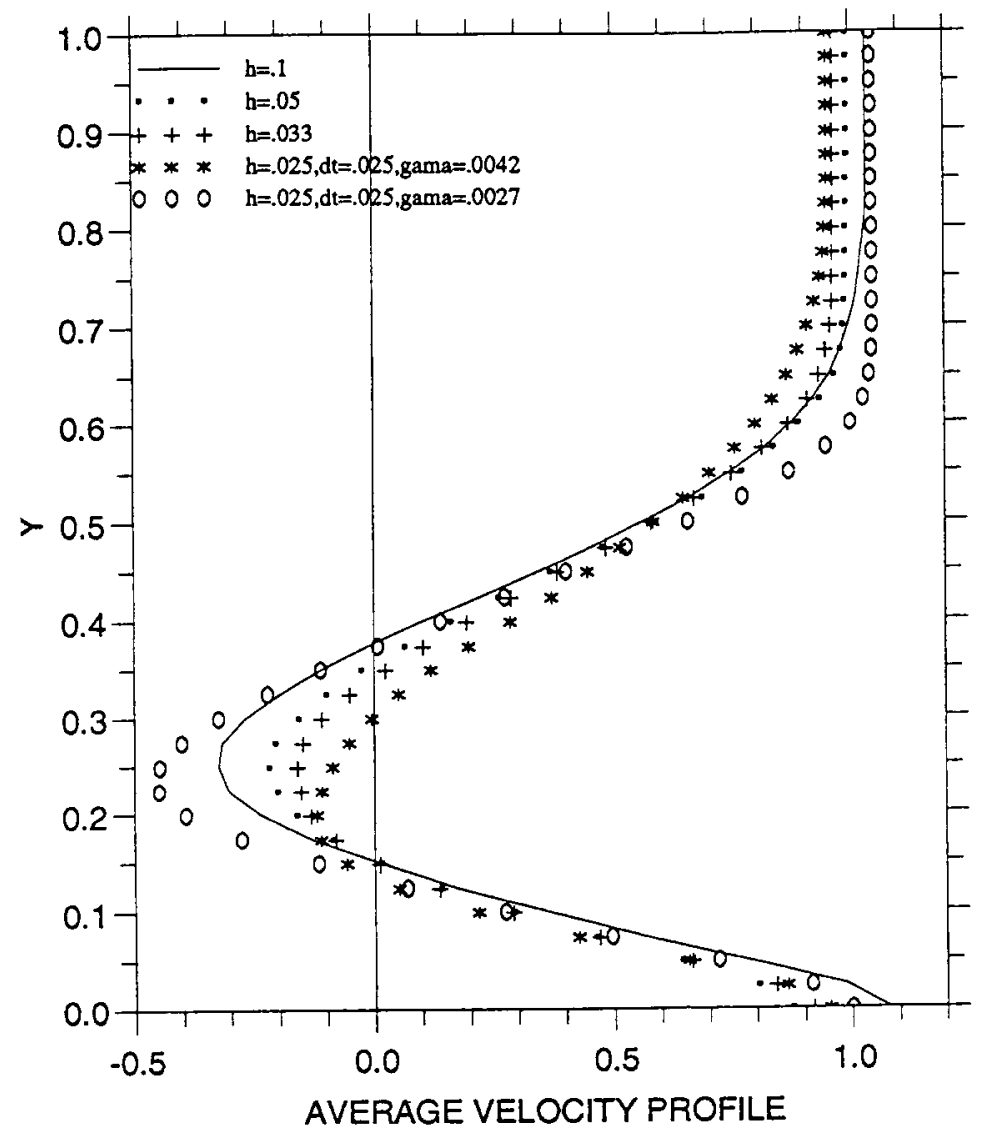

Figure 7: Average velocity profiles for $x=2 . \quad(R e=7000, \Gamma=0.00417, \Delta t=0.05$, for different values of $h$ ). 


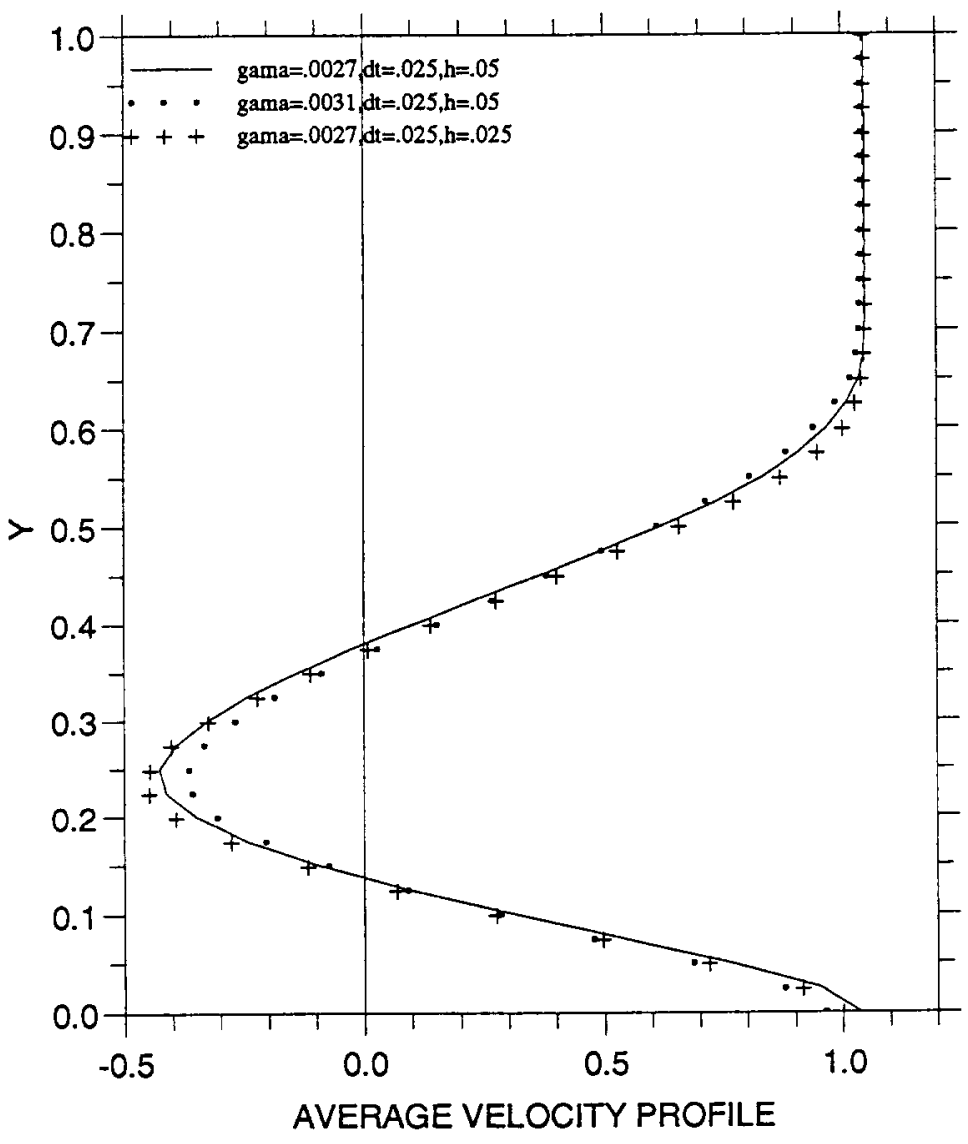

Figure 8: Average velocity profiles for $x=2$, with a combined choice of parameters. 